Intuitionistic fuzzy theory and its application in economy, technology and management

\title{
AN APPROACH FOR MADM PROBLEMS WITH INTERVAL-VALUED INTUITIONISTIC FUZZY SETS BASED ON NONLINEAR FUNCTIONS
}

\author{
Jian WU, Qingwei CAO, Hui LI \\ School of Economics and Management, Zhejiang Normal University, \\ Box 62 at YingBinDaDao 688, Jinhua 321004, Zhejiang Province, PR China
}

Received 06 November 2012; accepted 29 September 2013

\begin{abstract}
This paper investigates an approach for multiple attribute decision making (MADM) problems with interval-valued intuitionistic fuzzy numbers (IVIFNs). To do that, the nonlinear score, accuracy and hesitation functions of IVIFNs are developed based on the normal distribution. The novelty of these nonlinear functions is that they have an additional variance value, which can have more information to rank IVIFNs than Xu and Chen's score function and Ye's accuracy function. Based on these nonlinear functions, a ranking method for IVIFNs is proposed. Furthermore, a nonlinearly optimized model is proposed to obtain attribute weights by integrating these nonlinear functions. Then, we develop an approach for interval-valued intuitionistic fuzzy MADM programs in which two cases are considered: the attribute weight information is known and particularly known. In the end, we apply the proposed approach to select green supplier.
\end{abstract}

Keywords: multi-attribute decision-making, interval-valued intuitionistic fuzzy set, score function, accuracy function, hesitation function, normal distribution.

JEL Classification: C021, C441, D81, N55.

\section{Introduction}

The theory of fuzzy sets (FSs) proposed by Zadeh (1965) is a powerful tool to deal with vagueness, whose basic component is only a membership function. Atanassov (1986) introduced the concept of intuitionistic fuzzy set (IFS) characterized by a membership function and a non-membership function, which is an extension of Zadeh's fuzzy sets. Later, Atanassov and Gargov (1989) introduced the concept of interval-valued intuitionistic fuzzy sets (IVIFSs) as a further generalization of that of IFSs. The intuitionistic and interval-valued intuitionistic fuzzy set theory has been applied to many different fields, such as multiplicative criteria

Corresponding author Jian Wu

E-mail: jyajian@163.com 
decision making with complete weight information of attribute (Zhang, Liu 2010; Jiang et al. 2011; Park et al. 2011; Wei 2011; Yang, Chiclana 2009, 2012), multiplicative attribute decision making with incomplete weight information of attribute (Wang et al. 2009a, 2011a, 2011b; Pei, Zheng 2012; Yue 2011a; Zhao et al. 2012), multiplicative attribute decision making with intuitionistic or interval-valued intuitionistic fuzzy preference relations (Gong et al. 2009, 2011; Wu, Chiclana 2012; Xu 2013), group decision making (Li 2007; Li et al. 2010; Chen, Yang 2011; Su et al. 2011; Yue 2011b; Wei et al. 2012; Xu 2012; Zeng 2013; Xia, Xu 2013), aggregating operators (Wei 2009, 2010; Li 2011; Liu 2011; Merigó 2011; Merigó, Gil-Lafuente 2011;Wu, Cao 2013; Wu 2015), supplier selection (Boran et al. 2009), virtual enterprise partner selection (Ye 2010), strategy selection (Wei, Merigó 2012). In the decision making with intuitionistic or interval-valued intuitionistic fuzzy numbers, one key issue that needs to be addressed is to rank IFNs and IVFNs. Xu and Chen (2007) defined the score function and accuracy function to IVIFNs environments, and then developed an approach to rank IVFNs. However, in some cases, these functions do not allow the proper discrimination between different IVFNs. To resolve this problem, this article aims to develop the nonlinear score, accuracy and hesitation functions of IVIFNs based on the normal distribution, and then investigate a novel ranking approach.

The ranking problem has been extensively studied for the case of fuzzy numbers (FNs). A widely used approach to rank FNs is to convert them into a representative crisp value, and perform the comparison on them (Yager 2004), which is also the common one used to rank IFNs and IVIFNs. Representative crisp values developed for IFNs and IVIFNs are known with the names of score degree and accuracy degree. By the membership and nonmembership functions, Chen and Tan (1994) developed a score function for IFSs, which was later improved by Hong and Choi (2000) with the addition of an accuracy function. Other score and accuracy functions had been proposed in (Li et al. 2007; Wang et al. 2009b). Xu and Chen (2007) extended the score function and accuracy function to IVIFNs environments. Later, Ye (2009) proposed a different accuracy function that he claimed solved some drawbacks associated to the accuracy function developed by Xu and Chen (2007). However, both accuracy functions were proved to be equivalent when ordering IVIFNs (Wang 2011), and therefore the drawbacks highlighted by Ye (2009) were not properly addressed. Wu and Chiclana (2014) investigated a risk attitudinal score and accuracy expected functions to rank IVIFNs. Recently, Lakshmana Gomathi Nayagam and Sivaraman (2012) claimed that a novel accuracy function is proposed to compare IVIFNs. However, this accuracy function can be proved to be a score function more than an accuracy function. Indeed, in some cases, these proposals do not allow the proper discrimination between different IVIFNs. One key reason is that there is no research on the rationality of these functions, which may lead to misuse them in the process of raking IVIFNs. Another key reason is that they are straight forward extensions of their respective proposals for the case of IFNs. However, IVIFNs are more complicate than IFNs because that their membership and non-membership functions are interval numbers, which are nonlinear functions and can not be compared directly. Therefore they are not rich enough to capture all the information contained in IVIFNs.

To resolve these problems, we firstly build some judgment criterions to study the rationality of score and accuracy functions. Secondly, we develop the nonlinear score, accuracy 
and hesitation functions of IVIFNs based on the normal distribution. Our nonlinear functions extends: (i) Xu and Chen's score function for IVIFNs.; and (ii) Ye's accuracy function for IVIFNs. Then, we give an order relation between IVIFNs. This method guarantees that it can give sufficient information about interval-valued intuitionistic fuzzy numbers based on the score values, variance values and accuracy values. Finally, an approach for MADM programs with IVIFNs is proposed in which two cases are considered: the attribute weight information is known and particularly known.

The rest of this paper is organized as follows. In Section 1, we introduce some basic concepts related to IVIFSs. Some propositions are proposed to study the rationality of score and accuracy functions. Section 2 proposes the normal distribution based score accuracy and hesitation functions. In Section 3, an optimization model is developed to determine the attribute weights based on the nonlinear functions. Section 4 proposes an approach for MADM programs with IVIFNs in which two cases are considered: the attribute weight information is known and particularly known. Section 5 gives an illustrative numerical example to verify the developed approach. Finally, in the last Section we draw our conclusions.

\section{Preliminaries}

We start this section by introducing some basic concepts related to interval-valued intuitionistic fuzzy sets, which will be used throughout this paper.

Atanassov and Gargov (1989) introduced the notion of interval-valued intuitionistic fuzzy set (IVIFS), which is characterized by a membership function and a non-membership function, whose values are intervals rather than exact numbers.

Definition 1 (IVIFS of Atanassov and Gargov (1989)). Let $D \in[0,1]$ be the set of all closed subintervals of the interval and $X$ be a universe of discourse. An interval-valued intuitionistic fuzzy set in $A$ over $X$ is an object having the form:

$$
A=\left\{<x, \tilde{\mu}_{A}(x), \tilde{v}_{A}(x)>\mid x \in X\right\}
$$

where

$$
\tilde{\mu}_{A} \rightarrow D \in[0,1], \quad \tilde{v}_{A}(x) \rightarrow D \in[0,1]
$$

with the condition $0 \leq \sup \tilde{\mu}_{A}(x)+\sup \tilde{v}_{A}(x) \leq 1, \forall x \in X$.

The intervals $\tilde{\mu}_{A}(x)$ and $\tilde{v}_{A}(x)$ denote, respectively, the membership function and the non-membership function of the element $x$ to the set $A$. Thus for each $x \in X, \tilde{\mu}_{A}(x)$ and $\tilde{\mathrm{v}}_{A}(x)$ are closed intervals and their lower and upper end points are, respectively, denoted by $\tilde{\mu}_{A L}(x), \tilde{\mu}_{A U}(x), \tilde{v}_{A L}(x)$ and $\tilde{v}_{A U}(x)$. We can denote by

where

$$
A=\left\{<x,\left[\tilde{\mu}_{A L}(x), \tilde{\mu}_{A U}(x)\right],\left[\tilde{v}_{A L}(x), \tilde{v}_{A U}(x)\right]>\mid x \in X\right\},
$$

$$
0 \leq \tilde{\mu}_{A U}(x)+, \tilde{v}_{A U}(x) \leq 1, \tilde{\mu}_{A L}(x) \geq 0, \tilde{v}_{A L}(x) \geq 0 .
$$


For each element $x$, we can compute the unknown degree (hesitancy degree) of an intervalvalued intuitionistic fuzzy interval of $x \in X$ in $\tilde{A}$ defined as follows:

$$
\begin{aligned}
\pi_{A(x)}= & 1-\tilde{\mu}_{\tilde{A}}(x)-\tilde{v}_{\tilde{A}}(x)= \\
& {\left[1-\tilde{\mu}_{A U}(x)-\tilde{v}_{A U}(x)\right],\left[1-\tilde{\mu}_{A L}(x)-\tilde{v}_{A L}(x)\right] . }
\end{aligned}
$$

We will denote the set of all the IVIFSs in X by IVIFS(X). For convenience, let $\tilde{\mu}_{A}(x)=[a, b], \tilde{v}_{A}(x)=[c, d]$, so $A=([a, b],[c, d])$.

$\mathrm{Xu}$ and Chen (2007) proposed the following score and accuracy functions associated to an IVIFN:

Definition 2 (Score function of $X u$ and Chen (2007)). Let $A=([a, b],[c, d])$ be an interval-valued intuitionistic fuzzy number, a score function $S$ of an interval-valued intuitionistic fuzzy value can be represented as follows:

$$
S(A)=\frac{a+b-c-d}{2}, S(A) \in[-1,1]
$$

to evaluate the degree of score of the interval-valued intuitionistic fuzzy value $A=([a, b],[c, d])$, where $S(A) \in[-1,1]$. The larger the value of $S(A)$, the more the degree of score of the interval-valued intuitionistic fuzzy value $A$.

Definition 3 (Accuracy function of Xu and Chen (2007)). Let $A=([a, b],[c, d])$ be an interval-valued intuitionistic fuzzy number, an accuracy function $H$ of an interval-valued intuitionistic fuzzy value can be represented as follows:

$$
H(A)=\frac{a+b+c+d}{2}, H(A) \in[0,1]
$$

to evaluate the degree of accuracy of the interval-valued intuitionistic fuzzy value $A=([a, b],[c, d])$, where $H(A) \in[0,1]$. The larger the value of $H(A)$, the more the degree of accuracy of the interval-valued intuitionistic fuzzy value $A$.

Ye (2009) proposed a different expression for the accuracy degree of an IVIFN that has the same range of values $[-1,1]$, than the score degree defined above:

Definition 4 (Accuracy function of $Y e(2009))$. Let $A=([a, b],[c, d])$ be an interval-valued intuitionistic fuzzy number, a novel accuracy function $M$ of an interval-valued intuitionistic fuzzy value can be represented as follows:

$$
M(A)=a+b-1+\frac{c+d}{2},
$$

where $M(A) \in[-1,1]$. The larger the value of $M(A)$, the more the degree of accuracy of the interval-valued intuitionistic fuzzy value $A$.

Lakshmana Gomathi Nayagam and Sivaraman (2012) developed a new accuracy function of IVIFSs as follows: 
Definition 5 (Accuracy function of Lakshmana Gomathi Nayagam and Sivaraman (2012)). Let $A=([a, b],[c, d])$ be an interval-valued intuitionistic fuzzy number, a novel accuracy function $L$ of an interval-valued intuitionistic fuzzy value can be represented as follows:

$$
L(A)=\frac{a+b-d(1-b)-c(1-a)}{2} .
$$

The larger the value of $L(A)$, the more the degree of accuracy of the interval-valued intuitionistic fuzzy value $A$.

These functions have been universally used in decision making problems with IVIFNs. However, in some cases, these proposals do not allow the proper discrimination between different IVIFNs.

Example 1. If interval-valued intuitionistic fuzzy values for two alternatives are $\tilde{\alpha}_{1}=([0.05,0.35],[0.2,0.5])$ and $\tilde{\alpha}_{2}=([0.1,0.3],[0.3,0.4])$, then the desirable alternative is selected in accordance with accuracy function.

By applying Definition 2 and Definition 3, we can obtain $S\left(\tilde{a}_{1}\right)=S\left(\tilde{a}_{2}\right)=-0.15$ and $H\left(\tilde{a}_{1}\right)=H\left(\tilde{a}_{2}\right)=0.55$, respectively. In this case we do not know which alternative is better. By applying Definition 4 , we have $M\left(\tilde{a}_{1}\right)=-0.25$ and $M\left(\tilde{a}_{2}\right)=-0.25$. In both the cases we do not know which alternative is better. But, according to Definition 5 , we get $L\left(\tilde{a}_{1}\right)=0.015$ and $L\left(\tilde{a}_{2}\right)=-0.08$, and hence $\tilde{a}_{1}$ is better than $\tilde{a}_{2}$.

Example 2. If interval-valued intuitionistic fuzzy values for two alternatives are $\tilde{\alpha}_{1}=([0.2,0.2],[0.3,0.5])$ and $\tilde{\alpha}_{2}=([0.2,0.2],[0.1,0.7])$, then the desirable alternative is selected in accordance with accuracy function.

According to Definition 2 and Definition 3, we can obtain $S\left(\tilde{a}_{1}\right)=S\left(\tilde{a}_{2}\right)=-0.2$ and $H\left(\tilde{a}_{1}\right)=H\left(\tilde{a}_{2}\right)=0.6$, respectively. In this case we do not know which alternative is better. According to Definition 4 , we have $M\left(\tilde{a}_{1}\right)=M\left(\tilde{a}_{2}\right)=-0.2$. By Definition 5 , we get $L\left(\tilde{a}_{1}\right)=-0.12$ and $L\left(\tilde{a}_{2}\right)=-0.12$. In these three cases, we do not know which alternative is better.

The above examples demonstrate the limitation of these functions. However, the reasons for this limitation have not been discovered and discussed. To study the properties of score function and accuracy function, we propose the following propositions.

Proposition 1 (Monotonicity of score function). Let $A=([a, b],[c, d])$ be an interval-valued intuitionistic fuzzy number, the score function $S(A)=(a+b-c-d) / 2$ is a monotone increasing function with $a$ and $b$, and a monotone decreasing function with $c$ and $d$.

Proof. Omited.

Proposition 2 (Symmetry of score function). Let $A_{1}=\left(\left[a_{1}, b_{1}\right],\left[c_{1}, d_{1}\right]\right)$ and $A_{2}=\left(\left[a_{2}, b_{2}\right],\left[c_{2}, d_{2}\right]\right)$ be two IVIFNs, $\bar{A}_{1}=\left(\left[c_{1}, d_{1}\right],\left[a_{1}, b_{1}\right]\right)$ and $\bar{A}_{2}=\left(\left[c_{2}, d_{2}\right],\left[a_{2}, b_{2}\right]\right)$ be their associated inverse functions, receptively, then we have the following conclusion $S\left(A_{1}\right) \leq S\left(A_{2}\right) \Leftrightarrow S\left(\bar{A}_{1}\right) \geq S\left(\bar{A}_{2}\right)$.

Proof. (Sufficiency) From Definition 2, we obtain:

$$
S\left(A_{1}\right)=\frac{a_{1}+b_{1}-c_{1}-d_{1}}{2} \text { and } S\left(A_{2}\right)=\frac{a_{2}+b_{2}-c_{2}-d_{2}}{2}
$$


Since $S\left(A_{1}\right) \leq S\left(A_{2}\right)$, then

$$
a_{1}+b_{1}-c_{1}-d_{1} \leq a_{2}+b_{2}-c_{2}-d_{2} .
$$

That is:

$$
c_{1}+d_{1}-a_{1}-b_{1} \leq c_{2}+d_{2}-a_{2}-b_{2} \text {. }
$$

Thus, we have:

$$
S\left(\bar{A}_{1}\right)=\frac{c_{1}+d_{1}-a_{1}-b_{1}}{2} \leq \frac{c_{2}+d_{2}-a_{2}-b_{2}}{2}=S\left(\bar{A}_{2}\right) .
$$

We also can get the proof of necessity. The proof of Proposition 2 is completed.

Proposition 3 (Monotonicity of accuracy function). Let $A=([a, b],[c, d])$ be an intervalvalued intuitionistic fuzzy number, the accuracy functions $H(A), M(A)$ and $L(A)$ are the monotone increasing functions with $a, b, c$ and $d$.

Proof. Omited.

Proposition 4 (Symmetry of accuracy function). Let $A_{1}=\left(\left[a_{1}, b_{1}\right],\left[c_{1}, d_{1}\right]\right)$ be an IVIFN and $\bar{A}_{1}=\left(\left[c_{1}, d_{1}\right],\left[a_{1}, b_{1}\right]\right)$ be its associated inverse function, then we have the following conclusions 1) $\left.H\left(A_{1}\right)=H\left(\bar{A}_{1}\right), 2\right) M\left(A_{1}\right) \neq M\left(\bar{A}_{1}\right)$, and 3) $L\left(A_{1}\right) \neq L\left(\bar{A}_{1}\right)$.

Proof. 1) According the Definition 3, we have:

$$
H\left(A_{1}\right)=\frac{a_{1}+b_{1}+c_{1}+d_{1}}{2}=H\left(\bar{A}_{1}\right) .
$$

2) According the Definition 4, we obtain:

$$
M\left(A_{1}\right)=a_{1}+b_{1}+\frac{c_{1}+d_{1}}{2}-1,
$$

and

then,

$$
M\left(\bar{A}_{1}\right)=c_{1}+d_{1}+\frac{a_{1}+b_{1}}{2}-1
$$

$$
M\left(A_{1}\right) \neq M\left(\bar{A}_{1}\right) .
$$

3) According the Definition 5, we obtain:

$$
L\left(A_{1}\right)=\frac{a_{1}+b_{1}-d_{1}\left(1-b_{1}\right)-c_{1}\left(1-a_{1}\right)}{2}=\frac{a_{1}+b_{1}-d_{1}-c_{1}}{2}+\frac{a_{1} c_{1}+b_{1} d_{1}}{2}=S\left(A_{1}\right)+\frac{a_{1} c_{1}+b_{1} d_{1}}{2},
$$

and

$$
L\left(\bar{A}_{1}\right)=\frac{c_{1}+d_{1}-b_{1}\left(1-d_{1}\right)-a_{1}\left(1-c_{1}\right)}{2}=\frac{c_{1}+d_{1}-b_{1}-a_{1}}{2}+\frac{a_{1} c_{1}+b_{1} d_{1}}{2}=\frac{a_{1} c_{1}+b_{1} d_{1}}{2}-S\left(A_{1}\right),
$$

then

$$
L\left(A_{1}\right) \neq L\left(\bar{A}_{1}\right)
$$

Note 1. The accuracy functions $M(A)$ of Ye (2009) and $L(A)$ of Lakshmana Gomathi Nayagam and Sivaraman (2012) do not satisfy the Symmetry property. Moreover, we can prove that $L(A)$ satisfy the Monotonicity and Symmetry properties of score function. Thus, $L(A)$ is a score function more than an accuracy function. 


\section{Nonlinear functions of IVIFNs based on the normal distribution}

IVIFNs are more complicate than IFNs because that the membership and non-membership functions of the former are interval numbers. It is an established fact that interval numbers are nonlinear functions and can not be compared directly (Bortolan, Degani 1985). Therefore, the score and accuracy functions can not be the straight forward extensions of their respective proposals for the case of IFNs, which are linear functions. Considering that the numbers within the interval sometimes do not mean the same for decision makers, Ahn (2006) assumed that they are distributed by the normal distribution. Motivated by this idea, this article will propose some new score and accuracy functions for ranking IVIFNs. The novelty of these functions is that they are normally distributed.

Definition 6 (Normal distribution). Let $x(x \in[a, b])$ be the continuous random variable, and then we define its probability density function as:

$$
f(x)=\frac{1}{\sqrt{2 \pi \sigma}} e^{-\left[(x-u)^{2} / 2 \sigma^{2}\right]},
$$

where, the mean and variance of the normal distribution can be assumed to be $u=(a+b) / 2$ and $\sigma^{2}=(b-a)^{2} / 4$, respectively.

Consequently, for any interval-valued intuitionistic fuzzy number $A=([a, b],[c, d])$, its membership function and non-membership function can be approximated by the normal distribution, where $\tilde{\mu}_{A}(x) \sim N\left((a+b) / 2,(b-a)^{2} / 4\right)$ and $\tilde{v}_{A}(x) \sim N\left((c+d) / 2,(d-c)^{2} / 4\right)$, respectively.

Definition 7 (Normal distribution based score function). Let $A=([a, b],[c, d])$ be an interval-valued intuitionistic fuzzy number, a score function $\tilde{S}$ of $A$ can be represented as follows:

$$
\tilde{S}=\tilde{\mu}_{A}(x)-\tilde{v}_{A}(x) \sim N\left((a+b-c-d) / 2,\left[(b-a)^{2}+(d-c)^{2}\right] / 4\right),
$$

where the mean and variance of $\tilde{S}$ are $u_{\tilde{S}}=(a+b-c-d) / 2$ and $\sigma_{\tilde{S}}^{2}=\left[(b-a)^{2}+(d-c)^{2}\right] / 4$, respectively. The larger the value of $u_{\tilde{S}}$, the more the score degree of the interval-valued intuitionistic fuzzy value $\tilde{S}$.

Theorem 1. Let $A_{i}=\left(\left[a_{i}, b_{i}\right],\left[c_{i}, d_{i}\right]\right)(i=1,2, \ldots, m)$ be $m$ interval-valued intuitionistic fuzzy numbers and $\tilde{S}_{i}(i=1,2, \ldots, m)$ be their associated score functions. Then, the addition of these independent interval numbers $\tilde{S}_{i}(i=1,2, \ldots, m)$, each of which is normally distributed, is also normally distributed with a mean of $u_{\tilde{S}_{i}}$ and a variance of $\sigma_{\tilde{S}}^{2}$ :

$$
w_{1} \tilde{S}_{1}+w_{2} \tilde{S}_{2}+\cdots+w_{m} \tilde{S}_{m} \sim N\left(u, \sigma^{2}\right),
$$

with

$$
\begin{gathered}
u=w_{1} u_{\tilde{S}_{1}}+w_{2} u_{\tilde{S}_{2}}+\cdots+w_{m} u_{\tilde{S}_{m}}=\sum_{i=1}^{m} w_{i} u_{\tilde{S}_{i}} ; \\
\sigma^{2}=w_{1}^{2} \sigma_{\tilde{S}_{1}}^{2}+w_{2}^{2} \sigma_{\tilde{S}_{2}}^{2}+\cdots+w_{m}^{2} \sigma_{\tilde{S}_{m}}^{2}=\sum_{i=1}^{m} w_{i}^{2} \sigma_{\tilde{S}_{i}}^{2} .
\end{gathered}
$$


Proof: Since $\tilde{S}_{i}(i=1,2, \ldots, m)$ is normally distributed, we can obtain

then

$$
\tilde{S}_{i} \sim N\left(u_{\tilde{S}_{i}}, \sigma_{\tilde{S}_{i}}^{2}\right) \text {, }
$$

thus

$$
w_{i} \tilde{S}_{i} \sim N\left(w_{i} u_{\tilde{S}_{i}}, w_{i}^{2} \sigma_{\tilde{S}_{i}}^{2}\right)
$$

$$
w_{1} \tilde{S}_{1}+w_{2} \tilde{S}_{2}+\cdots+w_{m} \tilde{S}_{m} \sim N\left(\sum_{i=1}^{m} w_{i} u_{\tilde{S}_{i}}, \sum_{i=1}^{m} w_{i}^{2} \sigma_{\tilde{S}_{i}}^{2}\right)
$$

which has completed the proof of Theorem 1 .

Definition 8 (Normal distribution based accuracy function). Let $A=([a, b],[c, d])$ be an interval-valued intuitionistic fuzzy number, an accuracy function $\tilde{H}$ of $A$ can be represented as follows:

$$
\tilde{H}=\tilde{\mu}_{A}(x)+\tilde{v}_{A}(x) \sim N\left((a+b+c+d) / 2,\left[(b-a)^{2}+(d-c)^{2}\right] / 4\right),
$$

where the mean and variance of $\tilde{H}$ are $u_{\tilde{H}}=(a+b+c+d) / 2$ and $\sigma_{\tilde{H}}^{2}=\left[(b-a)^{2}+(d-c)^{2}\right] / 4$, respectively. The larger the value of $u_{\tilde{H}}$, the more the accuracy degree of the interval-valued intuitionistic fuzzy value $\tilde{H}$.

Theorem 2. Let $A_{i}=\left(\left[a_{i}, b_{i}\right],\left[c_{i}, d_{i}\right]\right)(i=1,2, \ldots, m)$ be $m$ interval-valued intuitionistic fuzzy numbers and $\tilde{H}_{i}(i=1,2, \ldots, m)$ be their associated accuracy functions. Then, the addition of these independent interval numbers $\tilde{H}_{i}(i=1,2, \ldots, m)$, each of which is normally distributed, is also normally distributed with a mean of $u_{\tilde{H}_{i}}$ and a variance of $\sigma_{\tilde{H}_{i}}^{2}$ :

with

$$
w_{1} \tilde{H}_{1}+w_{2} \tilde{H}_{2}+\cdots+w_{m} \tilde{H}_{m} \sim N\left(\bar{u}, \bar{\sigma}^{2}\right) \text {, }
$$

$$
\begin{gathered}
\bar{u}=w_{1} u_{\tilde{H}_{1}}+w_{2} u_{\tilde{H}_{2}}+\cdots+w_{m} u_{\tilde{H}_{m}}=\sum_{i=1}^{m} w_{i} u_{\tilde{H}_{i}} ; \\
\bar{\sigma}^{2}=w_{1}^{2} \sigma_{\tilde{H}_{1}}^{2}+w_{2}^{2} \sigma_{\tilde{H}_{2}}^{2}+\cdots+w_{m}^{2} \sigma_{\tilde{H}_{m}}^{2}=\sum_{i=1}^{m} w_{i}^{2} \sigma_{\tilde{H}_{i}}^{2} .
\end{gathered}
$$

Proof: Since $\tilde{H}_{i}(i=1,2, \ldots, m)$ is normally distributed, we can obtain:

then

$$
\tilde{H}_{i} \sim N\left(u_{\tilde{H}_{i}}, \sigma_{\tilde{H}_{i}}^{2}\right),
$$

$$
w_{i} \tilde{H}_{i} \sim N\left(w_{i} u_{\tilde{H}_{i}}, w_{i}^{2} \sigma_{\tilde{H}_{i}}^{2}\right)
$$

thus

$$
w_{1} \tilde{H}_{1}+w_{2} \tilde{H}_{2}+\cdots+w_{m} \tilde{H}_{m} \sim N\left(\sum_{i=1}^{m} w_{i} u_{\tilde{H}_{i}}, \sum_{i=1}^{m} w_{i}^{2} \sigma_{\tilde{H}_{i}}^{2}\right),
$$

which has completed the proof of Theorem 2.

Definition 9 (Normal distribution based hesitation function). Let $A=([a, b],[c, d])$ be an interval-valued intuitionistic fuzzy number, a hesitation function $\tilde{\pi}$ of $A$ can be represented as follows:

$$
\tilde{\pi}=1-\tilde{\mu}_{A}(x)-\tilde{v}_{A}(x) \sim N\left((2-a-b-c-d) / 2, \quad\left[(b-a)^{2}+(d-c)^{2}\right] / 4\right),
$$

where the mean and variance of $\tilde{\pi}$ are $u_{\tilde{\pi}}=(2-a-b-c-d) / 2$ and $\sigma_{\tilde{\pi}}^{2}=\left[(b-a)^{2}+(d-c)^{2}\right] / 4$, respectively. The larger the value of $u_{\tilde{\pi}}$, the more the hesitation degree of the interval-valued 
intuitionistic fuzzy value $\tilde{\pi}$. The larger the value of $\sigma_{\tilde{\pi}}^{2}$, the smaller the hesitation degree of the interval-valued intuitionistic fuzzy value $\tilde{\pi}$.

Theorem 3. Let $A_{i}=\left(\left[a_{i}, b_{i}\right],\left[c_{i}, d_{i}\right]\right)(i=1,2, \ldots, m)$ be $m$ interval-valued intuitionistic fuzzy numbers and $\tilde{\pi}_{i}(i=1,2, \ldots, m)$ be their associated hesitation function. Then, the addition of these independent interval numbers $\tilde{\pi}_{i}(i=1,2, \ldots, m)$, each of which is normally distributed, is also normally distributed with a mean of $\overline{\bar{u}}$ and a variance of $\overline{\bar{\sigma}}_{2}$ :

with

$$
w_{1} \tilde{\pi}_{1}+w_{2} \tilde{\pi}_{2}+\cdots+w_{m} \tilde{\pi}_{m} \sim N\left(\overline{\bar{u}}, \overline{\bar{\sigma}}^{2}\right),
$$

$$
\begin{gathered}
\overline{\bar{u}}=w_{1} u_{\tilde{\pi}_{1}}+w_{2} u_{\tilde{\pi}_{2}}+\cdots+w_{m} u_{\tilde{\pi}_{m}}=\sum_{i=1}^{m} w_{i} u_{\tilde{\pi}_{i}} ; \\
\overline{\bar{\sigma}}^{2}=w_{1}^{2} \sigma_{\tilde{\pi}_{1}}^{2}+w_{2}^{2} \sigma_{\tilde{\pi}_{2}}^{2}+\cdots+w_{m}^{2} \sigma_{\tilde{\pi}_{m}}^{2}=\sum_{i=1}^{m} w_{i}^{2} \sigma_{\tilde{\pi}_{i}}^{2} .
\end{gathered}
$$

Proof: Since $\tilde{\pi}_{i}(i=1,2, \ldots, m)$ is normally distributed, we can obtain:

then

$$
\tilde{\pi}_{i} \sim N\left(u_{\tilde{\pi}_{i}}, \sigma_{\tilde{\pi}_{i}}^{2}\right)
$$

$$
w_{i} \tilde{\pi}_{i} \sim N\left(w_{i} u_{\tilde{\pi}_{i}}, w_{i}^{2} \sigma_{\tilde{\pi}_{i}}^{2}\right)
$$

thus

$$
w_{1} \tilde{\pi}_{1}+w_{2} \tilde{\pi}_{2}+\cdots+w_{m} \tilde{\pi}_{m} \sim N\left(\sum_{i=1}^{m} w_{i} u_{\tilde{\pi}_{i}}, \sum_{i=1}^{m} w_{i}^{2} \sigma_{\tilde{\pi}_{i}}^{2}\right),
$$

which has completed the proof of Theorem 3.

Based on these three types of values for IVIFNs: the score function $S$, the accuracy function $H$ and hesitation function $\pi$, we shall present a method for the comparison between any two IVIFNs as follows:

Definition 10 (Order relation of IVIFNs). Let $\tilde{a}_{1}=\left(\left[a_{1}, b_{1}\right],\left[c_{1}, d_{1}\right]\right)$ and $\tilde{a}_{2}=\left(\left[a_{2}, b_{2}\right],\left[c_{2}, d_{2}\right]\right)$ be two interval-valued intuitionistic fuzzy values, $\tilde{S}\left(\tilde{a}_{1}\right) \sim N\left(\left(a_{1}+b_{1}-c_{1}-d_{1}\right) / 2\right.$, $\left[\left(b_{1}-a_{1}\right)^{2}+\right.$ $\left.\left(d_{1}-c_{1}\right)^{2}\right] / 4$ and $\tilde{S}\left(\tilde{a}_{2}\right) \sim N\left(\left(a_{2}+b_{2}-c_{2}-d_{2}\right) / 2,\left[\left(b_{2}-a_{2}\right)^{2}+\left(d_{2}-c_{2}\right)^{2}\right] / 4\right.$ be their associate score functions, respectively, and let $H\left(\tilde{a}_{1}\right) \sim N\left(\left(a_{1}+b_{1}+c_{1}+d_{1}\right) / 2,\left[\left(b_{1}-a_{1}\right)^{2}+\left(d_{1}-c_{1}\right)^{2}\right] / 4\right)$ and $H\left(\tilde{a}_{2}\right) \sim N\left(\left(a_{2}+b_{2}+c_{2}+d_{2}\right) / 2, \quad\left[\left(b_{2}-a_{2}\right)^{2}+\left(d_{2}-c_{2}\right)^{2}\right] / 4\right)$ be their associate accuracy functions, respectively, and let $\pi\left(\tilde{a}_{1}\right) \sim N\left(\left(1-a_{1}-b_{1}-c_{1}-d_{1}\right) / 2,\left[\left(b_{1}-a_{1}\right)^{2}+\left(d_{1}-c_{1}\right)^{2}\right] / 4\right)$ and $\pi\left(\tilde{a}_{2}\right) \sim N\left(\left(1-a_{2}-b_{2}-c_{2}-d_{2}\right) / 2,\left[\left(b_{2}-a_{2}\right)^{2}+\left(d_{2}-c_{2}\right)^{2}\right] / 4\right)$ be their associatehesitation functions, respectively, then:

(1) If $u_{S\left(\tilde{a}_{1}\right)}<u_{S\left(\tilde{a}_{2}\right)}$, then $\tilde{a}_{1}$ is smaller than $\tilde{a}_{2}$, denoted by $\tilde{a}_{1}<\tilde{a}_{2}$;

(2) If $u_{S\left(\tilde{a}_{1}\right)}=u_{S\left(\tilde{a}_{2}\right)}$, and, then if $u_{H\left(\tilde{a}_{1}\right)}<u_{H\left(\tilde{a}_{2}\right)}, \tilde{a}_{1}$ is smaller than $\tilde{a}_{2}$, denoted by $\tilde{a}_{1}<\tilde{a}_{2}$;

(3) If $u_{S\left(\tilde{a}_{1}\right)}=u_{S\left(\tilde{a}_{2}\right)}$, and $u_{H\left(\tilde{a}_{1}\right)}=u_{H\left(\tilde{a}_{2}\right)}$, then if $\sigma_{\pi\left(\tilde{a}_{1}\right)}^{2}<\sigma_{\pi\left(\tilde{a}_{2}\right)}^{2}, \tilde{a}_{1}$ is smaller than $\tilde{a}_{2}$, denoted by $\tilde{a}_{1}<\tilde{a}_{2}$;

(4) If $u_{S\left(\tilde{a}_{1}\right)}=u_{S\left(\tilde{a}_{2}\right)}, u_{H\left(\tilde{a}_{1}\right)}=u_{H\left(\tilde{a}_{2}\right)}$, and $\sigma_{\pi\left(\tilde{a}_{1}\right)}^{2}=\sigma_{\pi\left(\tilde{a}_{2}\right)}^{2}$, then $\tilde{a}_{1}$ and $\tilde{a}_{2}$ represent the same information, denoted $\tilde{a}_{1}=\tilde{a}_{2}$. 
In the following, we use the above approach to compare two IVIFMS $\tilde{a}_{1}$ and $\tilde{a}_{2}$ in Example 1 and Example 2, which shows that our functions are more reasonable than the functions proposed in (Xu, Chen 2007; Ye 2009; Lakshmana Gomathi Nayagam, Sivaraman 2012).

Example 3. (Example 1 continuation)

By Dentitions 7 and 8, we can obtain that $u_{S\left(\tilde{a}_{1}\right)}=u_{S\left(\tilde{a}_{2}\right)}=-0.15$ and $u_{\tilde{H}\left(\tilde{a}_{1}\right)}=u_{\tilde{H}\left(\tilde{a}_{2}\right)}=0.55$, respectively. Therefore, we can not use these values to compare $\tilde{a}_{1}$ and $\tilde{a}_{2}$. However, according to Defintion 9, we calculate the variance values of hesitation inedx $\sigma_{\pi\left(\tilde{a}_{1}\right)}^{2}=0.045$ and $\sigma_{\pi\left(\tilde{a}_{2}\right)}^{2}=0.0125$, respectively. According to Eq. (3) in Definition 10, we can get that $\tilde{a}_{1}$ is better than $\tilde{a}_{2}$.

Note 2: All of Definitions 2, 3 and 4 fail to rank interval-valued intuitionistic fuzzy values for two alternatives in this example. However, our approach is applicable.

Example 4. (Example 2 continuation)

According to Dentitions 7 and 8 , we get that $u_{S\left(\tilde{a}_{1}\right)}=u_{S\left(\tilde{a}_{2}\right)}=-0.2$ and $u_{H\left(\tilde{a}_{1}\right)}=u_{H\left(\tilde{a}_{2}\right)}=0.6$. There is no difference in $\tilde{a}_{1}$ and $\tilde{a}_{2}$ based on these two functions. However, by Dentition 9 , we obtain the variance value of hesitation index $\sigma_{\pi\left(\tilde{a}_{1}\right)}^{2}=0.01$ and $\sigma_{\pi\left(\tilde{a}_{2}\right)}^{2}=0.09$, respectively. According to Eq. (3) in Definition 10, we can get that $\tilde{a}_{2}$ is better than $\tilde{a}_{1}$.

Note 3: All of Definitions 2, 3 and 4 fail to rank interval-valued intuitionistic fuzzy values for two alternatives in this example. However, our approach is still applicable.

In the above two examples, our approach can rank these interval-valued intuitionistic fuzzy sets correctly. The advantage of our approach is the use of the variance value of hesitation index, which can provide more information for interval-valued intuitionistic fuzzy numbers.

\section{An optimization model for attribute weight based on nonlinear functions}

In some decision making problems, due to the increasing complexity of many practical decision situations, the DM may not be confident in providing exact values for attribute weights. Instead, the decision maker (DM) may only possess partial knowledge about attribute weights. The types of $Q$ provided by group members are linearly unequal constraints, which can be constructed by the following forms (Kim, Ahn 1999):

(1) A weak ranking: $\left\{w_{i} \geq w_{j}\right\}$;

(2) A strict ranking: $\left\{w_{i}-w_{j} \geq a_{i}\right\}$;

(3) A ranking with multiples: $\left\{w_{i} \geq a_{i} w_{j}\right\}$;

(4) An interval form: $\left\{a_{i} \leq w_{i} \leq a_{i}+\varepsilon_{i}\right\}$;

(5) A ranking of differences: $\left\{w_{i}-w_{j} \geq w_{k}-w_{l}\right\}$, for $j \neq k \neq l$,

where $\alpha_{i}$ and $\varepsilon_{i}$ are nonnegative constants.

From Eq. (8), we know that the score of alternative $x_{i}$ is based on the value of $S_{i}=\sum_{i=1}^{n} w_{i} \tilde{S}_{i j}$. Obviously, the greater the value $S_{i}$, the better the alternative $x_{i}$. Therefore, we can build the following three optimization models to drive attribute weight: 
346 J. Wu et al. An approach for MADM problems with interval-valued intuitionistic fuzzy sets...

$$
\begin{gathered}
\operatorname{Max} S_{i}=\sum_{i=1}^{n} w_{i} \tilde{S}_{i j} \\
\text { s.t. }\left\{\begin{array}{l}
W \in Q \\
\sum_{i=1}^{n} w_{i}=1 \\
0 \leq w_{i}
\end{array}\right.
\end{gathered}
$$

and

$$
\begin{gathered}
\operatorname{Max} H_{i}=\sum_{i=1}^{n} w_{i} \tilde{H}_{i j} \\
\text { s.t. }\left\{\begin{array}{l}
W \in Q \\
\sum_{i=1}^{n} w_{i}=1 \\
0 \leq w_{i}
\end{array}\right.
\end{gathered}
$$

and

$$
\begin{aligned}
\operatorname{Max} \sigma_{\tilde{S}_{i}}^{2} & =\sum_{i=1}^{n} \sum_{j=1}^{m} w_{i}^{2} \sigma_{\tilde{S}_{i j}}^{2} \\
\text { s.t. } & \left\{\begin{array}{l}
W \in Q \\
\sum_{i=1}^{n} w_{i}=1 \\
0 \leq w_{i}
\end{array} .\right.
\end{aligned}
$$

Since all of the alternatives in the MADM problems are competitive, the above multi-objective programming models could be further aggregated into a single objective programming as follows:

and

$$
\begin{aligned}
\operatorname{Max} S & =\sum_{i=1}^{n} \sum_{j=1}^{m} w_{i} u_{\tilde{S}_{i j}} \\
\text { s.t. } & \left\{\begin{array}{l}
W \in Q \\
\sum_{i=1}^{n} w_{i}=1 \\
0 \leq w_{i}
\end{array}\right.
\end{aligned}
$$

$$
\begin{aligned}
\operatorname{Max} \tilde{H} & =\sum_{i=1}^{n} \sum_{j=1}^{m} w_{i} u_{\tilde{H}_{i j}} \\
\text { s.t. } & \left\{\begin{array}{l}
W \in Q \\
\sum_{i=1}^{n} w_{i}=1 \\
0 \leq w_{i}
\end{array}\right.
\end{aligned}
$$


and

$$
\begin{aligned}
\operatorname{Max} \sigma_{\tilde{S}}^{2} & =\sum_{i=1}^{n} \sum_{j=1}^{m} w_{i}^{2} \sigma_{\tilde{S}}^{2} \\
\text { s.t. } & \left\{\begin{array}{l}
W \in Q \\
\sum_{i=1}^{n} w_{i}=1 \\
0 \leq w_{i}
\end{array} .\right.
\end{aligned}
$$

Because expressions (21), (22) and (23) are there maximization problems with the same constraints, they can be combined to formulate the following optimization program:

$$
\begin{aligned}
\operatorname{Max} Z & =\sum_{i=1}^{n} \sum_{j=1}^{m} w_{i} u_{\tilde{S}_{i j}}+w_{i} u_{\tilde{H}_{i j}}+w_{i}^{2} \sigma_{\tilde{H}_{i j}}^{2} \\
\text { s.t. } & \left\{\begin{array}{l}
W \in Q \\
\sum_{i=1}^{n} w_{i}=1 \\
0 \leq w_{i}
\end{array}\right.
\end{aligned}
$$

By resolving the above optimization program, we can obtain an optimal weight vector $W^{T}$.

\section{Multi-criteria fuzzy decision-making method based on the new novel score function and accuracy function}

In this section, we shall present an approach for Multi-criteria fuzzy decision-making problems with interval-valued intuitionistic fuzzy numbers based on the new novel score function and accuracy function.

Let $A=\left\{A_{1}, A_{2}, \ldots, A_{m}\right\}$ be a set of alternatives and let $C=\left\{C_{1}, C_{2}, \ldots, C_{m}\right\}$ be a set of criteria. Assume that the weight of the criterion $C_{j}(j=1,2, \ldots, n)$, entered by the decision-maker, is $w_{j}, w_{j} \in[0,1]$ and $\sum_{j=1}^{n} w_{j}=1$. In this case, the characteristic of the alternative $A_{i}$ is represented by an IVIFS:

$$
A_{i}=\left\{\left\langle C_{j},\left[\mu_{i L}\left(C_{j}\right), \mu_{i U}\left(C_{j}\right)\right],\left[v_{i L}\left(C_{j}\right), v_{i U}\left(C_{j}\right)\right]\right\rangle \mid C_{j} \in C\right\},
$$

where $0 \leq \mu_{i U}\left(C_{j}\right)+v_{i U}\left(C_{j}\right) \leq 1, \mu_{i L}\left(C_{j}\right) \geq 0, v_{i L}\left(C_{j}\right) \geq 0, j=1,2, \ldots, n$ and $i=1,2, \ldots, m$. The IVIFS value that is the pair of intervals $\mu_{A_{i}}\left(C_{j}\right)=\left[a_{i j}, b_{i j}\right], v_{A_{i}}\left(C_{j}\right)=\left[c_{i j}, d_{i j}\right]$ for $C_{j} \in C$ is denoted by $\alpha_{i j}=\left(\left[a_{i j}, b_{i j}\right],\left[c_{i j}, d_{i j}\right]\right)$, where $\left[a_{i j}, b_{i j}\right]$ indicates the degree that the alternative $A_{i}$ satisfies the criterion $C_{j}$ given by the decision maker, $\left[c_{i j}, d_{i j}\right]$ indicates the degree that the alternative $A_{i}$ does not satisfy the criterion $C_{j}$ given by the decision maker, $\left[a_{i j}, b_{i j}\right] \in[0,1],\left[c_{i j}, d_{i j}\right] \in[0,1]$. Therefore, we can elicit a decision matrix $D=\left(\alpha_{i j}\right)_{m \times n}$. The next four steps can summarize the procedure of applying this method. 
Case 1. The DMs have complete weight information.

Step 1. By Eq. (5), we calculate the score matrix $S=\left(s_{i j}\right)_{m \times n}$ of $D=\left(\alpha_{i j}\right)_{m \times n}$.

Step 2. Calculate the score values, accuracy values and variance values of interval-valued intuitionistic fuzzy value $\alpha_{i j}(i=1,2, \ldots, m, j=1,2, \ldots, n)$ by using Eq. (5) and Eq. (11).

Step 3. Aggregate the score values, accuracy values and variance values by using Eq. (6) and Eq. (10), respectively.

Step 4. Rank the alternative $A=\left\{A_{1}, A_{2}, \ldots, A_{m}\right\}$ and select the best one(s) according to Definition 9.

Step 5. End.

Case 2. The DMs have partial weight information.

Step 1. By resolve the Expression (24), we obtain an optimal weight vector $W^{T}$.

Step 2. By Eq. (5), we calculate the score matrix $S=\left(s_{i j}\right)_{m \times n}$ of $D=\left(\alpha_{i j}\right)_{m \times n}$.

Step 3. Calculate the score values, accuracy values and variance values of interval-valued intuitionistic fuzzy value $\alpha_{i j}(i=1,2, \ldots, m i=1,2, \ldots, m, j=1,2, \ldots, n)$ by using Eq. (5) and Eq. (11).

Step 4. Aggregate the score values, accuracy values and variance values by using Eq. (6) and Eq. (10), respectively.

Step 5. Rank the alternative $A=\left\{A_{1}, A_{2}, \ldots, A_{m}\right\}$ and select the best one(s) according to Definition 9.

Step 6. End.

\section{Illustrative example}

With increasing governmental regulation and stronger public awareness in environmental protection, environmental performance evaluation has become an important issue in green production. An electronic company is desirable to select its green suppliers. After pre-evaluation, four suppliers. $A_{i}(i=1,2,3,4) A_{i}(i=1,2,3,4)$ are remained as alternatives for further evaluation. Four criteria are considered as: $C_{1}$ : Remanufacturing activity; $C_{2}$ : Energy consumption; $C_{3}$ : Hazardous waste management; $C_{4}$ : Environmental certification. Since most of these criteria are qualitative, there exist some fuzziness and uncertainty in this type of decision making problem. Therefore, the assessments by interval-valued intuitionistic fuzzy numbers to four alternatives are shown in Table 1.

Table 1. Assessments of four green suppliers based on each criterion

\begin{tabular}{cllll}
\hline \multirow{2}{*}{ Suppliers } & \multicolumn{3}{c}{ Criteria } \\
\cline { 2 - 5 } & \multicolumn{1}{c}{$C_{1}$} & \multicolumn{1}{c}{$C_{2}$} & \multicolumn{1}{c}{$C_{4}$} \\
\hline \multirow{2}{*}{$A_{1}$} & $([0.40,0.65],[0.10$, & $([0.30,0.50],[0.10$, & $([0.50,0.60],[0.20$, & $([0.60,0.70],[0.20$, \\
& $0.30])$ & $0.20])$ & $0.40])$ & $0.30])$ \\
\hline \multirow{2}{*}{$A_{2}$} & $([0.50,0.75],[0.10$, & $([0.50,0.60],[0.20$, & $([0.45,0.55],[0.35$, & $([0.20,0.30],[0.10$, \\
& $0.20])$ & $0.30])$ & $0.45])$ & $0.20])$ \\
\hline \multirow{2}{*}{$A_{3}$} & $([0.30,0.70],[0.10$, & $([0.40,0.70],[0.10$, & $([0.40,0.60],[0.15$, & $([0.50,0.60],[0.30$, \\
& $0.20])$ & $0.20])$ & $0.40])$ & $0.40])$ \\
\hline \multirow{2}{*}{$A_{4}$} & $([0.40,0.70],[0.30$, & $([0.60,0.80],[0.10$, & $([0.30,0.50],[0.10$, & $([0.20,0.50],[0.10$, \\
& $0.30])$ & $0.20])$ & $0.20])$ & $0.40])$ \\
\hline
\end{tabular}


From Table 1, we can get the following decision making matrix:

$$
D=\left[\begin{array}{llll}
([0.40,0.65],[0.1,0.3]) & ([0.3,0.5],[0.1,0.2]) & ([0.50,0.60],[0.20,0.40]) & ([0.6,0.7],[0.2,0.3]) \\
([0.50,0.75],[0.1,0.2]) & ([0.5,0.6],[0.2,0.3]) & ([0.45,0.55],[0.35,0.45]) & ([0.2,0.3],[0.1,0.2]) \\
([0.30,0.70],[0.1,0.2]) & ([0.4,0.7],[0.1,0.2]) & ([0.40,0.60],[0.15,0.40]) & ([0.5,0.6],[0.3,0.4]) \\
([0.40,0.70],[0.3,0.3]) & ([0.6,0.8],[0.1,0.2]) & ([0.30,0.50],[0.10,0.20]) & ([0.2,0.5],[0.1,0.4])
\end{array}\right] .
$$

\subsection{The DMs have complete weight information}

Assume that the weights of $C_{1}, C_{2}, C_{3}$ and $C_{4}$ are $0.4,0.3,0.1$ and 0.2 , respectively. Then, we utilize our approach to get the most desirable alternative(s).

Step 1. According to expression (1), we calculate the associated score values of $s_{i j}$ $(i=1,2, \ldots, m, j=1,2, \ldots, n)$ in Table 2 .

Table 2. The score values of $s_{i j}$

\begin{tabular}{ccccc}
\hline \multirow{2}{*}{$\begin{array}{c}\text { Score } \\
\text { values }\end{array}$} & $C_{1}$ & $C_{2}$ & $C_{3}$ & $C_{4}$ \\
\hline$A_{1}$ & 0.325 & 0.250 & 0.250 & 0.400 \\
\hline$A_{2}$ & 0.475 & 0.300 & 0.100 & 0.100 \\
\hline$A_{3}$ & 0.250 & 0.400 & 0.225 & 0.200 \\
\hline$A_{4}$ & 0.250 & 0.550 & 0.250 & 0.100 \\
\hline
\end{tabular}

Using the weights vector of criteria $W=(0.4,0.3,0.1,0.2)^{T}$, we obtain the overall score values $S_{i}(i=1,2,3,4)$ of the alternative $A_{i}$ as follows:

$$
S_{1}=0.3100, S_{2}=0.3100, S_{3}=0.3225, S_{4}=0.3100 .
$$

Then, we have that $A_{3} \succ A_{4}=A_{1}=A_{2}$. Obviously, the score function of $\mathrm{Xu}$ and Chen (2007) is not able to rank the alternatives $A_{1}, A_{2}$ and $A_{4}$. In the following, we further rank these there alternatives. To do that, we compute the accuracy values $s_{i j}(i=1,2, \ldots, m$, $j=1,2, \ldots, n)$ in Table 3 .

Table 3. The accuracy values of $s_{i j}$

\begin{tabular}{ccccc}
\hline \multirow{2}{*}{$\begin{array}{c}\text { Accuracy } \\
\text { values }\end{array}$} & $C_{1}$ & $C_{2}$ & $C_{3}$ & $C_{4}$ \\
\cline { 2 - 5 } & 0.725 & 0.550 & 0.850 & 0.900 \\
\hline$A_{1}$ & 0.775 & 0.800 & 0.900 & 0.400 \\
\hline$A_{2}$ & 0.650 & 0.700 & 0.775 & 0.900 \\
\hline$A_{3}$ & 0.850 & 0.850 & 0.550 & 0.600 \\
\hline$A_{4}$ & & &
\end{tabular}

By applying the weights vector of criteria $W=(0.4,0.3,0.1,0.2)^{T}$, we calculate the overall accuracy values $H_{i}(i=1,2,3,4)$ of the alternative $A_{i}$ as follows:

$$
H_{1}=0.7200, H_{2}=0.7200, H_{3}=0.7275, H_{4}=0.7700 \text {. }
$$


According to Definition 10, we obtain that $A_{3} \succ A_{4} \succ A_{1}=A_{2}$. However, the accuracy function of $\mathrm{Xu}$ and Chen (2007) is still not able to rank the alternatives $A_{1}$ and $A_{2}$. In the following, we compute the variance values of $s_{i j}(i=1,2, \ldots, m, j=1,2, \ldots, n)$ in Table 4 .

Table 4. The variance values of $s_{i j}$

\begin{tabular}{ccccc}
\hline \multirow{2}{*}{$\begin{array}{c}\text { Variance } \\
\text { values }\end{array}$} & $C_{1}$ & $C_{2}$ & $C_{3}$ & $C_{4}$ \\
\cline { 2 - 5 } & 0.0256 & 0.0125 & 0.0125 & 0.0050 \\
\hline$A_{1}$ & 0.0181 & 0.0050 & 0.0050 & 0.0050 \\
\hline$A_{2}$ & 0.0425 & 0.0250 & 0.0256 & 0.0050 \\
\hline$A_{3}$ & 0.0225 & 0.0125 & 0.0125 & 0.0450 \\
\hline$A_{4}$ & & & &
\end{tabular}

By expression (17), we calculate the overall variance values $\sigma_{S_{i}}^{2}(i=1,2,3,4)$ of the alternative $A_{i}$ as follows:

$$
\sigma_{S_{1}}^{2}=0.0055, \sigma_{S_{2}}^{2}=0.0036, \sigma_{S_{3}}^{2}=0.0095, \sigma_{S_{4}}^{2}=0.0066
$$

Then, we have $A_{1} \succ A_{2}$. According to Definition 10, we have the ranking order of the alternatives: $A_{3} \succ A_{4} \succ A_{1} \succ A_{2}$. Consequently, the variance value $\sigma_{S_{i}}^{2}$ is a useful tool when the score values and accuracy functions do not allow the proper discrimination between different interval-valued intuitionistic fuzzy numbers.

To further study the properties of the score functions, accuracy functions, and variance functions, we compute the accuracy values of Ye (2009) in Table 5.

Table 5. Ye's accuracy values of $s_{i j}$

\begin{tabular}{ccccc}
\hline \multirow{2}{*}{$M_{i j}$} & \multicolumn{3}{c}{ Criteria } \\
\cline { 2 - 5 } & $C_{1}$ & $C_{2}$ & $C_{3}$ & $C_{4}$ \\
\hline$A_{1}$ & 0.250 & -0.050 & 0.400 & 0.550 \\
\hline$A_{2}$ & 0.400 & 0.350 & 0.400 & -0.350 \\
\hline$A_{3}$ & 0.150 & 0.250 & 0.275 & 0.450 \\
\hline$A_{4}$ & 0.400 & 0.550 & -0.050 & -0.050 \\
\hline
\end{tabular}

By expression (3), we obtain Ye's overall accuracy values $M_{i}(i=1,2,3,4)$ of the alternative $A_{i}$ as follows:

$$
M_{1}=0.2350, M_{2}=0.2350, M_{3}=0.2525, M_{4}=0.3100 .
$$

Then we obtain that $A_{4} \succ A_{3} \succ A_{1}=A_{2}$, where $A_{1}$ and $A_{2}$ are not discriminated.

We also can calculate the accuracy function values of Lakshmana Gomathi Nayagam and Sivaraman (2012) in Table 6. 
Table 6. Nayagam's accuracy values of $s_{i j}$

\begin{tabular}{ccccc}
\hline \multirow{2}{*}{$L A_{i j}$} & $C_{1}$ & $C_{2}$ & $C_{3}$ & $C_{4}$ \\
\cline { 2 - 5 } & 0.885 & 0.630 & 0.840 & 1.130 \\
\hline$A_{1}$ & 1.150 & 0.880 & 0.605 & 0.280 \\
\hline$A_{2}$ & 0.870 & 0.980 & 0.750 & 0.790 \\
\hline$A_{3}$ & 0.830 & 1.320 & 0.630 & 0.420 \\
\hline$A_{4}$ & & & &
\end{tabular}

By Definition 5, we obtain Nayagam's overall accuracy values $L A_{i}(i=1,2,3,4)$ of the alternative $A_{i}$ as follows:

$$
L A_{1}=0.8530, L A_{2}=0.8405, L A_{3}=0.8750, L A_{4}=0.8750,
$$

Then, we obtain that $A_{3}=A_{4} \succ A_{1} \succ A_{2}$. This ranking order is similar as the one by our proposed approach, which demonstrates $L A$ is a score function more than an accuracy function. However, $A_{3}$ and $A_{4}$ are still not discriminated in this case.

\subsection{The DMs have partial weight information}

Step 1. The information about the attribute weights is partly known as follows:

$$
Q=\left\{0.1 \leq w_{1} \leq 0.3,0.2 \leq w_{2} \leq 0.4,0.15 \leq w_{3} \leq 0.3,0.28 \leq w_{4} \leq 0.4, \sum_{j=1}^{n} w_{n}=1,0 \leq w_{i} \leq 1\right\} .
$$

Based on the expression (24), we build the following optimization model:

$$
\begin{aligned}
\operatorname{Max} Z & =\sum_{i=1}^{n} \sum_{j=1}^{m} 4.4 w_{1}+4.4 w_{2}+3.9 w_{3}+3.6 w_{4}+0.1 w_{1}^{2}+0.06 w_{2}^{2}+0.055 w_{3}^{2}+0.06 w_{4}^{2} \\
& \left\{\begin{array}{l}
0.1 \leq w_{1} \leq 0.3 \\
0.2 \leq w_{2} \leq 0.4 \\
0.15 \leq w_{3} \leq 0.3 \\
0.28 \leq w_{4} \leq 0.4 \\
w_{1}+w_{2}+w_{3}+w_{4}=1 \\
0 \leq w_{i} \leq 1
\end{array}\right.
\end{aligned}
$$

By resolving this model, we obtain the weight vector.

Step 2. By using Eq. (7) and Eq. (8), we can obtain weighed values of each alternative as follows:

Step 3. According to Definition 10, we rank all alternatives: . And then, the most desirable alternative is.

Step 4. End.

\subsection{Analysis of the nonlinear functions}

The ranking method for interval-valued intuitionistic fuzzy numbers proposed in this paper has the following main advantages with respect to other methods proposed in the literature: 
352 J. Wu et al. An approach for MADM problems with interval-valued intuitionistic fuzzy sets...

1. It builds some judgment criterions to study the rationality for score and accuracy functions, It is worth mentioning that this issue that has not been successfully addressed.

2. It studies some desirable properties of score and accuracy functions: Monotonicity and Symmetry.

3. It supports the decision making progress in which the information weights about attribute is partly known, i.e. it presents a nonlinearly optimized model to obtain the weights of attributes.

Finally, the ranking method proposed in this paper differs with respect to the existing models (Xu, Chen 2007; Ye 2009; Lakshmana Gomathi Nayagam, Sivaraman 2012) in the following aspects:

1. It allows the presence of the nonlinear score and accuracy functions, which are based on the normal distribution.

2. It ranks interval-valued intuitionistic fuzzy numbers by incorporating score function, accuracy function, and variance function. Therefore, it has more information than $\mathrm{Xu}$ and Chen's score function and Ye's accuracy function.

3. It proves thatof Lakshmana Gomathi Nayagam and Sivaraman (2012) is a score function more than an accuracy function.

\section{Conclusions}

This article develops the nonlinear score, accuracy and hestitation functions of IVIFNs based on the normal distribution. Then, we study their desirable properties: Monotonicity and Symmetry. Based on these nonlinear functions, an approach for ranking interval-valued intuitionistic fuzzy numbers is proposed. The novelty of this approach is that it contains three values: the score values, variance values and accuracy values. As a result, it can give more information than $\mathrm{Xu}$ and Chen's score function and Ye's accuracy function. By combining these nonlinear functions, we investigate a multi-criterion decision-making method with IVIFNs in which two cases are considered: the attribute weight information is known and particularly known. Finally, an illustrative example is provided to illustrate our proposed approach. Considered that, sometimes, the interval numbers may follow other distribution, such as distribution. In our future work, we shall focus on the new functions of intervalvalued intuitionistic fuzzy numbers based on other distributions.

\section{Acknowledgements}

The authors are very grateful to Editors and the anonymous referees for their valuable comments and suggestions that have helped us to improve considerably the quality of this paper. This work was supported by National Natural Science Foundation of China (NSFC) under the Grant (No. 71571166 and No. 71101131), Zhejiang Provincial Natural Science Foundation of China (No. LY15G010003), Zhejiang Provincial National Science Foundation for Distinguished Young Scholars of China (No. LR13G010001), Zhejiang Provincial Key Research Base of Humanistic and Social Sciences in Hangzhou Dianzi University (No. ZD01-201502), 
Zhejiang Provincial Qianjiang Talent Foundation of China (No. QJC1402015) and Zhejiang

Provincial Social Science Association Foundation of China (2015Z026).

\section{References}

Ahn, B. S. 2006. The uncertain OWA aggregation with weighting functions having a constant level of orness, International Journal of Intelligent Systems 21: 469-483. http://dx.doi.org/10.1002/int.20144

Atanassov, K. 1986. Intuitionstic fuzzy sets, Fuzzy Sets and Systems 20: 87-96. http://dx.doi.org/10.1016/S0165-0114(86)80034-3

Atanassov, K.; Gargov, G. 1989. Interval-valued intuitionistic fuzzy sets, Fuzzy Sets and Systems 31: 343-349. http://dx.doi.org/10.1016/0165-0114(89)90205-4

Boran, F. E.; Genc, S.; Kurt, M.; Akay, D. 2009. A multi-criteria intuitionistic fuzzy group decision making for supplier selection with TOPSIS method, Expert Systems with Applications 36: 11363-11368. http://dx.doi.org/10.1016/j.eswa.2009.03.039

Bortolan, G.; Degani, R. 1985. A review of some for ranking fuzzy subsets, Fuzzy sets and systems 15: 21-31. http://dx.doi.org/10.1016/0165-0114(85)90012-0

Chen, S. M.; Tan, J. M. 1994. Handling multi-criteria fuzzy decision-making problems based on vague set theory, Fuzzy Sets and Systems 67(2): 163-172. http://dx.doi.org/10.1016/0165-0114(94)90084-1

Chen, Z. P.; Yang, W. 2011. A new multiple attribute group decision making method in intuitionistic fuzzy setting, Applied Mathematical Modelling 35: 4424-4437. http://dx.doi.org/10.1016/j.apm.2011.03.015

Gong, Z. W.; Li, L. S.; Zhou, F. X.; Yao, T. X. 2009. Goal programming approaches to obtain the priority vectors from the intuitionistic fuzzy preference relations, Computers and Industrial Engineering 57: 1187-1193. http://dx.doi.org/10.1016/j.cie.2009.05.007

Gong, Z. W.; Li, L. S.; Forrest, J.; Zhao, Y. 2011. The optimal priority models of the intuitionistic fuzzy preference relation and their application in selecting industries with higher meteorological sensitivity, Expert Systems with Applications 38: 4394-4402. http://dx.doi.org/10.1016/j.eswa.2010.09.109

Hong, D. H.; Choi, C. H. 2000. Multicriteria fuzzy decision-making problems based on vague set theory, Fuzzy Sets and Systems 114: 103-113. http://dx.doi.org/10.1016/S0165-0114(98)00271-1

Jiang, Y. C.; Tang, Y.; Chen, Q. M. 2011. An adjustable approach to intuitionistic fuzzy soft sets based decision making, Applied Mathematical Modelling 35: 824-836. http://dx.doi.org/10.1016/j.apm.2010.07.038

Kim, S.; Ahn, B. S. 1999. Interactive group decision making procedure under incomplete information, European Journal of Operational Research 116: 498-507. http://dx.doi.org/10.1016/S0377-2217(98)00040-X

Lakshmana Gomathi Nayagam, V.; Sivaraman, G. 2012. Ranking of interval-valued intuitionistic fuzzy sets, Applied Soft Computing 11: 3368-3372. http://dx.doi.org/10.1016/j.asoc.2011.01.008

Li, D. F. 2007. Compromise ratio method for fuzzy multi-attribute group decision making, Applied Soft Computing 7(3): 807-817. http://dx.doi.org/10.1016/j.asoc.2006.02.003

Li, D. F. 2011. The GOWA operator based approach to multiattribute decision making using intuitionistic fuzzy sets, Mathematical and Computer Modelling 53: 1182-1196. http://dx.doi.org/10.1016/j.mcm.2010.11.088

Li, D. F.; Chen, G. H.; Hua, Z.Q. 2010. Linear programming method for multiattribute group decision making using IF sets, Information Sciences 180: 1591-1609. http://dx.doi.org/10.1016/j.ins.2010.01.017

Li, L.; Yuan, X. H.; Xia, Z. Y. 2007. Multicriteria fuzzy decision-making methods based on intuitionistic fuzzy sets, Journal of Computer and System Sciences 73: 84-88.

http://dx.doi.org/10.1016/j.jcss.2006.03.004 
Liu, P. D. 2011. A weighted aggregation operators multi-attribute group decision-making method based on interval-valued trapezoidal fuzzy numbers, Expert Systems with Applications 38: 1053-1060. http://dx.doi.org/10.1016/j.eswa.2010.07.144

Merigó, J. M. 2011. Fuzzy multi-person decision making with fuzzy probabilistic aggregation operators, International Journal of Fuzzy Systems 13(3): 163-174.

Merigó, J. M.; Gil-Lafuente, A. M. 2011. Fuzzy induced generalized aggregation operators and its application in multi-person decision making, Expert Systems with Applications 38(8): 9761-9772. http://dx.doi.org/10.1016/j.eswa.2011.02.023

Park, J. H.; Park, Y.; Kwun, C. Y.; Tan, X. G. 2011. Extension of the TOPSIS method for decision making problems under interval-valued intuitionistic fuzzy environment, Applied Mathematical Modelling 35: 2544-2556. http://dx.doi.org/10.1016/j.apm.2010.11.025

Pei, Z.; Zheng, L. 2012. A novel approach to multi-attribute decision making based on intuitionistic fuzzy sets, Expert Systems with Applications 39: 2560-2566. http://dx.doi.org/10.1016/j.eswa.2011.08.108

Su, Z. X.; Chen, M. Y.; Xia, G. P.; Li, W. 2011. An interactive method for dynamic intuitionistic fuzzy multi- attribute group decision making, Expert Systems with Applications 38: 15286-15295. http://dx.doi.org/10.1016/j.eswa.2011.06.022

Wang, J. Q.; Meng, L. Y.; Chen, X. H. 2009a. Multi-criteria decision making method based on vague sets and risk attitudes of decision makers, Systems Engineering and Electronics 2: 361-365.

Wang, W. Z. 2011. Comments on "Multicriteria fuzzy decision-making method based on a novel accuracy function under interval-valued intuitionistic fuzzy environment" by Jun Ye, Expert Systems with Applications 38: 13186-13187. http://dx.doi.org/10.1016/j.eswa.2011.04.130

Wang, Z. J.; Li, K. W.; Wang, W. Z. 2009b. An approach to multiattribute decision making with intervalvalued intuitionistic fuzzy assessments and incomplete weights, Information Sciences 179(17): 3026-3040. http://dx.doi.org/10.1016/j.ins.2009.05.001

Wang, Z. J.; Li, K. W.; Xu, J. H. 2011a. A mathematical programming approach to multi-attribute decision making with interval-valued intuitionistic fuzzy assessment information, Expert Systems with Applications 38: 12462-12469. http://dx.doi.org/10.1016/j.eswa.2011.04.027

Wang, Z.; Xu, Z. S.; Liu, S. S; Tang, J. 2011b. A netting clustering analysis method under intuitionistic fuzzy environment, Applied Soft Computing 11: 5558-5564. http://dx.doi.org/10.1016/j.asoc.2011.05.004

Wei, G. W. 2009. Some geometric aggregation functions and their application to dynamic multiple attribute decision making in intuitionistic fuzzy setting, International Journal of Uncertainty, Fuzziness and Knowledge- Based Systems 17: 179-196. http://dx.doi.org/10.1142/S0218488509005802

Wei, G. W. 2010. Some induced geometric aggregation operators with intuitionistic fuzzy information and their application to group decision making, Applied Soft Computing 10: 423-431. http://dx.doi.org/10.1016/j.asoc.2009.08.009

Wei, G. W. 2011. Grey relational analysis method for intuitionistic fuzzy multiple attribute decision making, Expert Systems with Applications 38: 11671-11677. http://dx.doi.org/10.1016/j.eswa.2011.03.048

Wei, G. W.; Merigó, J. M. 2012. Methods for strategic decision making problems with immediate probabilities in intuitionistic fuzzy setting, Scientia Iranica 19(6): 1936-1946. http://dx.doi.org/10.1016/j.scient.2012.07.017

Wei, G. W.; Zhao, X. F.; Wang, H. J. 2012. An approach to multiple attribute group decision making with interval intuitionistic trapezoidal fuzzy information, Technological and Economic Development of Economy 18(2): 317-330. http://dx.doi.org/10.3846/20294913.2012.676995

Wu, J. 2015. A SD-IITFOWA operator and TOPSIS based approach for MAGDM problems with intuistionistic trapezoidal fuzzy numbers, Technological and Economic Development of Economy 21(1): 28-47. http://dx.doi.org/10.3846/20294913.2014.946982 
Wu, J.; Cao, Q. W. 2013. Same families of geometric aggregation operators with intuitionistic trapezoidal fuzzy numbers, Applied Mathematical Modelling 37: 318-327. http://dx.doi.org/10.1016/j.apm.2012.03.001

Wu, J.; Chiclana, F. 2012. Non-dominance and attitudinal prioritisation methods for intuitionistic and interval-valued intuitionistic fuzzy preference relations, Expert Systems with Applications 39: 13049-13416. http://dx.doi.org/10.1016/j.eswa.2012.05.062

$\mathrm{Wu}$, J; Chiclana, F. 2014. A risk attitudinal ranking method for interval-valued intuitionistic fuzzy numbers based on novel score and accuracy expected functions, Applied Soft Computing 22: 272-286. http://dx.doi.org/10.1016/j.asoc.2014.05.005

Xia, M. M.; Xu, Z. S. 2013. Group decision making based on intuitionistic multiplicative aggregation operators, Applied Mathematical Modelling 37: 5120-5133. http://dx.doi.org/10.1016/j.apm.2012.10.029

$\mathrm{Xu}, \mathrm{Z}$. S. 2012. Intuitionistic fuzzy multi-attribute decision making: an interactive method, IEEE Transcations on Fuzzy Systems 20(2): 514-525.

$\mathrm{Xu}, \mathrm{Z}$. S. 2013. Priority weight intervals derived from intuitionistic multiplicative preference relations, IEEE Transactions on Fuzzy System 21: 642-654. http://dx.doi.org/10.1109/TFUZZ.2012.2226893

$\mathrm{Xu}, \mathrm{Z}$. S.; Chen, J. 2007. An approach to group decision making based on interval-valued intuitionistic judgment matrices, System Engineer-Theory and Practice 27: 126-133. http://dx.doi.org/10.1016/S1874-8651(08)60026-5

Yager, R. R. 2004. OWA aggregation over a continuous interval argument with applications to decision making, IEEE Transactions on Systems, Man, and Cybernetics - Part B: Cybernetics 34(5): 1952-1963. http://dx.doi.org/10.1109/TSMCB.2004.831154

Yang, Y. J.; Chiclana, F. 2009. Intuitionistic fuzzy sets: spherical representation and distances, International Journal of Intelligent Systems 24(4): 399-420. http://dx.doi.org/10.1002/int.20342

Yang, Y. J.; Chiclana, F. 2012. Consistency of 2d and 3d distances of intuitionistic fuzzy sets, Expert Systems with Applications 39(10): 8665-8670. http://dx.doi.org/10.1016/j.eswa.2012.01.199

Ye, F. 2010. An extended TOPSIS method with interval-valued intuitionistic fuzzy numbers for virtual enterprise partner selection, Expert Systems with Applications 37: 7050-7055. http://dx.doi.org/10.1016/j.eswa.2010.03.013

Ye, J. 2009. Multicriteria fuzzy decision-making method based on a novel accuracy function under interval-valued intuitionistic fuzzy environment, Expert Systems with Applications 36: 6899-6902. http://dx.doi.org/10.1016/j.eswa.2008.08.042

Yue, Z. L. 2011a. An approach to aggregating interval numbers into interval-valued intuitionistic fuzzy information for group decision making, Expert Systems with Applications 38: 6333-6338. http://dx.doi.org/10.1016/j.eswa.2010.11.108

Yue, Z. L. 2011b. Developing a straightforward approach for group decision making based on determining weights of decision makers, Applied Mathematical Modelling 36: 4106-4117. http://dx.doi.org/10.1016/j.apm.2011.11.041

Zadeh, L. A. 1965. Fuzzy sets, Information and Control 8: 338-353. http://dx.doi.org/10.1016/S0019-9958(65)90241-X

Zeng, S. Z. 2013. Some intuitionistic fuzzy weighted distance measures and their application to group decision making, Group decision and Negotiation 22(2): 281-298. http://dx.doi.org/10.1007/s10726-011-9262-6

Zhang, X.; Liu, P. D. 2010. Method for aggregating triangular fuzzy intuitionistic fuzzy information and its application to decision making, Technological and Economic Development of Economy 16(2): 280-290. http://dx.doi.org/10.3846/tede.2010.18

Zhao, H.; Xu, Z. S.; Liu, S. S.; Wang, Z. 2012. Intuitionistic fuzzy MST clustering algorithms, Computers and Industrial Engineering 62: 1130-1140. http://dx.doi.org/10.1016/j.cie.2012.01.007 
Jian WU received the PhD degree in management science and engineering from Hefei University of Technology, Hefei, China, in 2008 and is currently an associated professor with School of Economics and Management, Zhejiang Normal University, China. He is an Associate Editor of the Journal of Intelligent and Fuzzy Systems and Guest Editor of the Journal of Applied Soft Computing. He is one of the FUZZ-IEEE2014 Program Committee Members and Co-chair of the special issue: Fuzzy decision-making: Consensus and Missing preferences. He has 30+ papers published in leading journals such as INS, CAIE, ASOC, KNOSYS, ESWA, IJIS, MCM, AMM, AMC, TEDE and ITOR. He was an academic research visitor in CCI, at De Montfort University.

Qingwei CAO is currently a lecture with School of Economics and Management, Zhejiang Normal University, China. Her current research interests include: group decision making, and aggregation operators. She has authored or coauthored several papers published in international journals such as CAIE, ESWA, MCM and APM.

Hui LI is a professor with School of Economics and Management, Zhejiang Normal University, China. His current research interests include Financial Decision Aiding and Accounting Information Systems; Case-Based Reasoning; Business Computing and Business Forecasting. He has 50+ papers published on leading and reputable journals, e.g.: European Journal of Operational Research, IEEE Transactions on Systems, Man and Cybernetics-Part A, Information \& Management, Information Sciences, International Journal of Systems Science, Expert Systems, Expert Systems with Applications, Journal of Forecasting, Knowledge-Based Systems, Applied Soft Computing, Computers \& Industrial Engineering, Computers \& Operations Research, Technological and Economic Development of Economy, Tourism Management, WSEAS Transactions on Systems, etc. 